\title{
MEASUREMENT SCALES WITH ARBITRARY UNITS AND "EXPERT" INTERPRETATION AND RELATIVE MEASUREMENT THROUGH COMPARISONS AND DERIVED PRIORITIES
}

\author{
Thomas 1. Saaty* \\ University of Pittsburgh \\ E-mail: $\underline{\text { saaty@katz.pitt.edu }}$
}

\begin{abstract}
In science generally we create or invent ratio scales for measurement. It is known that such scales are based on arbitrarily chosen units and are invariant under a positive similarity transformation. Formulas are then built that use these scales. In the end experts must interpret the results both of the measurements and of the formulas. If they disagree, there is no mathematical way to combine their judgments. In the AHP we derive relative priority scales from judgments based on meaning at the beginning using an absolute scale and pairwise comparisons. An absolute scale is invariant under the identity transformation and is a special case of a ratio scale with multiplier equal to one. In each comparison the less dominant member of the pair is treated as the unit and an estimate is made as to how many times the other member dominates it. The number assigned to this dominance relation is of necessity an absolute number as it is invariant under the identity transformation. No other number can be used to represent the multiple than what it is, thus it is a number from an absolute scale. A relative scale is then derived from the pairwise comparisons. In the AHP these relative scales are normalized readings from an absolute scale. It is known that such a scale is not based on the choice of an arbitrary unit. If people differ in judgment, we can combine their judgments mathematically. It is surprising that there is a general form for the formulas derived from relative measurement.
\end{abstract}

\section{Introduction}

Our purpose here is to relate the continuous generalization of the AHP to physics formulas that are frequently determined empirically. Because the AHP generalization involves mathematically derived eigenfunctions based on judgments, we consider the outcome the most basic way to derive natural laws that is possible. We only have the Fourier transform of the real and complex solutions of our equation that is a necessary condition for the solvability of Fredholm's equation of a continuum of pairwise comparisons. We have Table 1:

\footnotetext{
${ }^{*}$ Corresponding author
} 
Table1 Solutions of the functional equation $w(a s)=b w(s)$

Proportionate Response to Natural Law

\begin{tabular}{|c|c|c|c|}
\hline $\begin{array}{l}\text { Kind of } \\
\text { Solution }\end{array}$ & Solution & Fourier Transform & Comments \\
\hline Real & $\begin{array}{l}w(s)= \\
b^{\log s / \log a} p\left(\frac{\log s}{\log a}\right) \\
\text { or more simply: } \\
w(u)=e^{\alpha u} p(u), \alpha=\log b, u=\log \\
s / \log a .\end{array}$ & $\begin{array}{l}\int_{-\infty}^{+\infty} \sum_{k=-\infty}^{\infty} \alpha_{k} \delta(\xi-2 \pi k) \frac{\beta}{\beta^{2}+(x-\xi)^{2}} d \xi \\
=\sum_{k=-\infty}^{\infty} \alpha_{k} \frac{\beta}{\beta^{2}+(x-2 k \pi)^{2}} \\
\text { If } P(u)=\cos u / 2 \pi=(1 / 2)\left(\mathrm{e}^{\mathrm{i} / 2 \pi}+\mathrm{e}^{-\mathrm{iu} / 2 \pi}\right) \\
\text { then: } \\
\text { C } \frac{\beta}{\sqrt{2 \pi}}\left[\frac{1}{\beta^{2}+\left(\frac{1}{2 \pi}+\xi\right)^{2}}+\frac{1}{\beta^{2}+\left(\frac{1}{2 \pi}-\xi\right)^{2}}\right]\end{array}$ & $\begin{array}{l}\text { The solution } \\
\text { of } \\
\text { Fredholm's } \\
\text { equation is } \\
\text { defined in } \\
\text { the } \\
\text { frequency } \\
\text { domain or } \\
\text { transform } \\
\text { domain in } \\
\text { Fourier } \\
\text { analysis as it } \\
\text { is based on } \\
\text { the flow of } \\
\text { electric } \\
\text { charge. }\end{array}$ \\
\hline $\begin{array}{l}\text { Complex: } \\
\text { General single } \\
\text { valued }\end{array}$ & $\begin{array}{l}w(z)=c b^{\log z /|\log g|} g(z) \text { where } \\
g(z) \text { is an arbitrary solution } \\
\text { of } g(a z)=g(z) \text {. }\end{array}$ & $\begin{array}{l}F(x)=\log a \sum_{n=-\infty}^{\infty} a_{n}^{\prime}\left(1 /(2 \pi i) \int_{-\infty}^{\infty} e^{[2 \pi n+\theta(b)] u u} e^{(\log a+\log \mid b) u} e^{-i z u} d u\right. \\
=(1 / 2 \pi) \log a \sum_{-\infty}^{\infty} a_{n}\left[\frac{(2 \pi n+\theta(b)-x)}{(\log a|b|+(2 \pi n+\theta(b)-x)} i\right] \\
\delta(2 \pi n+\theta(b)-x) .]\end{array}$ & \\
\hline Analytic & $\begin{array}{l}w(z)=\sum a^{\prime \prime}{ }_{j} z^{j+q}, \quad q<k \\
\text { The sum taken from } 0 \text { to } \\
\text { plus infinity if } w \text { is assumed } \\
\text { to be analytic everywhere, } \\
\text { but the sum is taken from } \\
\text { minus infinity to plus } \\
\text { infinity if } w \text { need not be } \\
\text { analytic at } 0 .\end{array}$ & $(2 \pi) \sum_{j=0}^{\infty}(i)^{-(j k+q)} a_{j}^{\prime} \frac{d^{j k+q} \delta(x)}{d x^{j k+q}}$ & \\
\hline Quaternions & & & \\
\hline
\end{tabular}


Octonions

Besides real and complex solutions, we also need solutions in quaternions and octonions because they form the remaining other two division algebras. We do not have them.

\section{How to Generate Simple Results in Physics}

\subsection{Discrete Judgments and the Inverse Square Law in Optics}

Below is an experiment to validate the relationship between the pairwise judgment and the Inverse Square Law in optics. Four identical chairs were placed on a line from a light source at the distances of 9, 15, 21, and 28 yards. The purpose was to see if one could stand by the light and look at the chair and compare their relative brightness in pairs, fill in the judgment matrix and obtain a relationship between the chairs and their distance from the light source. This experiment was repeated twice with different judges whose judgment matrices are shown in Table 2 .

Table 2 Pairwise Comparisons of the Four Chairs

$\begin{gathered}\text { Relative visual brightness } \\
\text { (1st Trial) }\end{gathered}$
\begin{tabular}{l|llll} 
& $C_{1}$ & $C_{2}$ & $C_{3}$ & $C_{4}$ \\
\hline$C_{1}$ & 1 & 5 & 6 & 7 \\
$C_{2}$ & $1 / 5$ & 1 & 4 & 6 \\
$C_{3}$ & $1 / 6$ & $1 / 4$ & 1 & 4 \\
$C_{4}$ & $1 / 7$ & $1 / 6$ & $1 / 4$ & 1
\end{tabular}

Relative visual brightness (2nd Trial)

The judges of the first matrix were the author's young children, ages 5 and 7 at that time, who gave their judgments qualitatively. The judge of the second matrix was the author's wife, also a mathematician not present during the children's judgment process. In Table 3 we give the principal eigenvectors, eigenvalues, consistency indices and consistency ratios of the two matrices.

Table 3 Principal Eigenvectors and Corresponding Measures

$\begin{array}{cc}\begin{array}{c}\text { Relative brightness eigenvector } \\ (1 \mathrm{st} \text { Trial) }\end{array} & \begin{array}{c}\text { Relative brightness eigenvector } \\ (2 \mathrm{nd} \text { Trial) }\end{array} \\ 0.61 & 0.62 \\ 0.24 & 0.22 \\ 0.10 & 0.10 \\ 0.05 & 0.06 \\ \lambda_{\max }=4.39, \text { C.I. }=0.13, \text { C.R. }=0.14 \lambda_{\max }=4.10, \text { C.I. }=0.03, \text { C.R. }=0.03\end{array}$

Table 3 Inverse Square Law of Optics 


\begin{tabular}{llllll} 
Distance & $\begin{array}{l}\text { Normalized } \\
\text { distance }\end{array}$ & $\begin{array}{l}\text { Square of } \\
\text { normalized } \\
\text { distance }\end{array}$ & $\begin{array}{l}\text { Reciprocal } \\
\text { of previous } \\
\text { column }\end{array}$ & $\begin{array}{l}\text { Normalized } \\
\text { reciprocal }\end{array}$ & $\begin{array}{l}\text { Rounding } \\
\text { off }\end{array}$ \\
\hline 9 & 0.123 & 0.015129 & 66.098 & 0.6079 & 0.61 \\
15 & 0.205 & 0.042025 & 23.79 & 0.2188 & 0.22 \\
21 & 0.288 & 0.082944 & 12.05 & 0.1108 & 0.11 \\
28 & 0.384 & 0.147456 & 6.78 & 0.0623 & 0.06 \\
\hline
\end{tabular}

First and second trial eigenvectors of Table 2 have been compared with the last column of Table 3 calculated from the inverse square law of optics. The root mean square deviations (RMS) and the median absolute deviation around the median (MAD) were computed and are both very small. It is interesting and important to observe that the judgments have captured a natural law here. It would seem that they could do the same in other areas of perception or thought, as we show in the next example.

Note the sensitivity of the results as the closest chair is moved even closer to the light source, for then it absorbs most of the value of the relative index and a small error in its distance from the source yields great error in the values. What is noteworthy from this sensory experiment is the observation or hypothesis that the observed intensity of illumination varies (approximately) inversely with the square of the distance. The more carefully designed the experiment, the better the results obtained from the visual observations.

\section{The General Inverse Squares Law}

We gave before the expression

$$
\int_{-\infty}^{+\infty} \sum_{k=-\infty}^{\infty} \alpha_{k} \delta(\xi-2 \pi k) \frac{\beta}{\beta^{2}+(x-\xi)^{2}} d \xi=\sum_{k=-\infty}^{\infty} \alpha_{k} \frac{\beta}{\beta^{2}+(x-2 k \pi)^{2}}
$$

We have already mentioned that this solution is general and is applicable to phenomena requiring relative measurement through ratio scales. We considered the case where

$$
P(u)=\cos u / 2 \pi=(1 / 2)\left(\mathrm{e}^{\mathrm{i} w / 2 \pi}+\mathrm{e}^{-\mathrm{i} u / 2 \pi}\right) .
$$

The inverse Fourier transform of $w(u)=C \mathrm{e}^{-\beta u} \cos u / 2 \pi, \beta>0$ is given by:

$$
C \frac{\beta}{\sqrt{2 \pi}}\left[\frac{1}{\beta^{2}+\left(\frac{1}{2 \pi}+\xi\right)^{2}}+\frac{1}{\beta^{2}+\left(\frac{1}{2 \pi}-\xi\right)^{2}}\right]
$$


When the constants in the denominator are small relative to $\xi$ we have $C_{1} / \xi^{2}$ which we believe is why optics, gravitation (Newton) and electric (Coulomb) forces act according to inverse square laws. This is the same law of nature in which an object responding to a force field must decide to follow that law by comparing infinitesimal successive states through which it passes. If the stimulus is constant, the exponential factor in the general response solution given in the last chapter is constant, and the solution in this particular case would be periodic of period one. When the distance $\xi$ is very small, the result varies inversely with the parameter $\beta>0$.

More importantly and very intriguing is the possibility that $\beta$ may be very large and its effect balances off the effect of $\xi$. Since a stimulus often passes through a medium and arrives somewhat weaker at its response destination, the effect of the medium may be great and affects the response substantially. It seems that dark matter in space acts like such a gravitational influence medium, and moderates behavior. For example it is well-known that the outer stars of a galaxy would be driven much farther due to centrifugal force and the effect of distance, but the effect of dark matter serves to pull these stars inwards, diminishing the overall effect of the forces countering gravitation.

\section{On the Convergence of the Solution in the Reals}

Here is a convergence condition for $\sum_{k=-\infty}^{\infty} a_{k}=\sum_{k=-\infty}^{+\infty} \alpha_{k} \frac{\beta}{\beta^{2}+(x-2 \pi k)^{2}}$.

Using the ratio test if $L=\lim _{k \rightarrow \infty}\left|\frac{a_{k+1}}{a_{k}}\right|<1$ the series $\sum_{k=-\infty}^{\infty} a_{k}$ converges.

Write $\sum_{k=-\infty}^{+\infty} \alpha_{k} \frac{\beta}{\beta^{2}+(x-2 \pi k)^{2}}=\sum_{k=-\infty}^{+\infty} \frac{\alpha_{k}}{\beta} \frac{1}{1+\left(\frac{x-2 \pi k}{\beta}\right)^{2}}$

$L=\lim _{k \rightarrow \infty}\left|\frac{a_{k+1}}{a_{k}}\right|=\lim _{k \rightarrow \infty}\left|\frac{\frac{\alpha_{k+1}}{\beta} \frac{1}{1+\left(\frac{x-2 \pi k-2 \pi}{\beta}\right)^{2}}}{\frac{\alpha_{k}}{\beta} \frac{1}{1+\left(\frac{x-2 \pi k}{\beta}\right)^{2}}}\right|=\lim _{k \rightarrow \infty}\left|\frac{\alpha_{k+1}}{\alpha_{k}}\right|\left|\frac{1+\left(\frac{x-2 \pi k}{\beta}\right)^{2}}{1+\left(\frac{x-2 \pi k-2 \pi}{\beta}\right)^{2}}\right|=\lim _{k \rightarrow \infty}\left|\frac{\alpha_{k+1}}{\alpha_{k}}\right|$

If $\lim _{k \rightarrow \infty}\left|\frac{\alpha_{k+1}}{\alpha_{k}}\right|<1$ the series converges. $\sum_{k=-\infty}^{+\infty} \alpha_{k} \frac{\beta}{\beta^{2}+(x-2 \pi k)^{2}} \leq \sum_{k=-\infty}^{+\infty} \frac{\alpha_{k}}{\beta}$.

\section{Formulas in Physics}


It is a considerable task to relate the foregoing along with its generalizations to the physical sciences. In general we have the following theories to look at in physics:

Kepler's laws, Newtonian mechanics and gravitation, the n-body problem, Maxwell's equations, Relativity, Quantum theory and Shrödinger's equation, String theory, astronomy.

In this note we will simply highlight a few equations that involve inverse squares on rewriting them. Like Newton's $F=m a=m d / t^{2}$ and Einstein's $E=m c^{2}$, or $m=E / c^{2}$.

Note that the above solution in the complex domain involves Dirac Delta distributions that are dense in very general spaces and at least in theory, can be used to approximate to any function. Here one needs to investigate the case of the Fourier Transform of several stimuli. It may be that a research workshop to deal with this very demanding task needs to be organized.

\section{REFERENCES}

Saaty, T. L., Principia Mathematica Decernendi: Mathematical Principles of Decision Making, RWS Publications, 4922 Ellsworth Avenue, Pittsburgh, PA 15213, 2010. 